\title{
iNOS Induces Vascular Endothelial Cell Migration and Apoptosis Via Autophagy in Ischemia/Reperfusion Injury
}

\author{
Tiebing Zhua,b Qi Yao ${ }^{a}$ Wei Wang ${ }^{a}$ Honghong Yaoc,d Jie Chao ${ }^{\text {b,d,e }}$ \\ aDepartment of Cardiology, The First Affiliated Hospital of Nanjing Medical University, ${ }^{b}$ Department of \\ Physiology, Medical School of Southeast University, 'Department of Pharmacology, School of Medicine, \\ Southeast University, 'Key Laboratory of Developmental Genes and Human Disease, Institute of Life

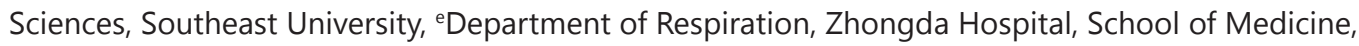 \\ Southeast University; Nanjing, China
}

\section{Key Words}

HUVEC • iNOS • Angiogenesis • Autophagy • Migration • Apoptosis

\begin{abstract}
Background/Aims: Inducible nitric oxide synthase (iNOS) plays a crucial role in ischemia/ reperfusion (I/R). Autophagy is involved in irreversible cell injury and death under extreme conditions. However, whether iNOS mediates myocardial ischemia/reperfusion (I/R) injury in endothelial cells via autophagy remains ill-defined. In this study, we examined whether I/Rmediated up-regulation of iNOS is critical in the modulation of cell migration and apoptosis via autophagy in human umbilical vein endothelial cells (HUVECs). Methods: iNOS expression was detected in HUVECs using Western blotting analyses and immunocytochemistry. An in vitro scratch assay was performed to detect cell migration. The autophagy markers ATG5, LC3B and BECN were detected using Western blotting analysis and adenovirus-mRFP-GFPLC3. The pharmacological inhibitor of autophagy 3-MA was also applied to confirm the role of autophagy in I/R. Results: I/R induced the expression of iNOS, which subsequently increased the migration and apoptosis of HUVECs and was associated with the up-regulation of autophagy. The iNOS specific inhibitor L-NAME abolished I/R-induced autophagy, while L-NAME and 3-MA both attenuated cell apoptosis and migration induced by I/R. Conclusion: These findings suggested that I/R-induced iNOS regulates migration and apoptosis in HUVECs via autophagy, which indicates a new therapeutic strategy for individuals with I/R injury.
\end{abstract}

(C) 2016 The Author(s)

Published by S. Karger AG, Basel

\section{Introduction}

Ischemia/reperfusion (I/R) injury is an important aspect of cardiovascular disease, which is the leading cause of death in advanced and industrialized countries [1-3]. Previous data from our lab and other studies have suggested that vascular endothelial cell dysfunction 
is an important issue in ischemic heart disease, which can result in cardiac remodeling and heart failure [4-6]. However, the mechanisms underlying this dysfunction remain poorly understood.

Abnormal angiogenesis is an important consequence of vascular endothelial cell dysfunction, which is involved in inflammatory diseases, such as diabetes and ischemic heart disease $[7,8]$. Inflammatory mediators are thought to play an important role in the generation of new blood vessels [5, 9]. Previous data from our lab have suggested that monocyte chemotactic protein-1 (MCP-1) and its downstream factor-MCP-1-induced protein (MCPIP1) are implicated in angiogenesis, which involves the activation and conversion of quiescent endothelial cells to migratory and proliferative phenotypes, as well as the differentiation of endothelial cells to an angiogenic phenotype [5, 10-13]. Endothelial cell apoptosis has recently been implicated in the initiation of angiogenesis and the regression of neo-vessels [14-16]. Inducible nitric oxide synthase (iNOS) expression has been associated with apoptosis in in vivo and in vitro disease models, in which macrophages and epithelial cells are mainly distributed around the inflammatory area, and its expression is induced by several stimuli [17-19]. Interestingly, recent studies have also suggested a correlation between MCP-1 and iNOS in different clinical settings [20-22]. For example, Chen et al., showed that baicalin ameliorates isoproterenol-induced acute myocardial infarction via iNOS and MCP-1 in rats [19]. Moreover, the expression of MCP-1 and iNOS induced by infection in epithelial cells was suppressed via NADPH oxidase and the Jak2/Stat3 pathway, which suggests an interaction between MCP-1 and iNOS [17]. However, the involvement of iNOS in the regulation of endothelial cell dysfunction and the mechanisms by which iNOS mediates this process remain unclear.

Autophagy plays a key role in the maintenance of cell homeostasis via the degradation of misfolded or dysfunctional proteins and organelles such as peroxisomes and mitochondria [23-25]. However, autophagy is also involved in irreversible cell injury and cell death under extreme conditions [26-30]. Furthermore, the up-regulation of iNOS has been shown to cause autophagy malfunction [31]. For instance, gentamicin increases the expression of iNOS, which induces inflammation, apoptosis, autophagy, and finally, nephrotoxicity in rats [32]. However, whether iNOS regulates endothelial cell dysfunction via autophagy remains unclear.

The present study aimed to determine the effect of I/R injury on the expression of iNOS in human umbilical vein endothelial cells (HUVECs) as well as the effect of iNOS on I/R-induced cellular migration and apoptosis and, particularly, the role of autophagy in this process. The results and conclusions obtained in this study may aid clinicians and researchers in understanding the regulation of iNOS expression and its functional relevance to I/R injury and provide insight into potential therapeutic targets for the treatment of myocardial ischemia.

\section{Materials and Methods}

Reagents

Fetal bovine serum (FBS), normal goat serum, Dulbecco's modified Eagle's medium (DMEM; \#1200046), and 10X-MEM (11430-030) were all obtained from Life Technologies. Amphotericin B (BP2645) and GlutaMax Supplement (35050-061) were obtained from Gibco, and Pen Strep (15140-122) was obtained from Fisher Scientific. PureCol type I bovine collagen ( $3 \mathrm{mg} / \mathrm{mL}$ ) was obtained from Advanced Biomatrix. Antibodies for p53 (SC6243, rabbit), MCPIP1 (SC136750, goat) and $\beta$-actin (SC8432, mouse) were obtained from Santa Cruz Biotechnology, Inc. The transfection reagent for the short-interfering RNA (siRNA), DharmaFECT, was purchased from Thermo Scientific. HUVECs were purchase from Genomeditech.

Cell culture

HUVECs were purchased from ScienCell ${ }^{\circledR}$ and maintained in T75 flasks containing DMEM with $10 \%$ FBS. HUVECs at passage 3-5 (P3-5) were stored in liquid nitrogen. A vial of P3-5 HUVECs was thawed, 


\section{Cellular Physiology Cell Physiol Biochem 2016;38:1575-1588 \begin{tabular}{l|l} 
and Biochemistry Published online: April 18, 2016 & $\begin{array}{l}\text { D } 2016 \text { The Author(s). Published by S. Karger AG, Basel } \\
\text { www.karger.com/cpb }\end{array}$
\end{tabular} \\ Zhu et al.: iNOS Activates HUVECs via Autophagy in I/R}

plated, and passaged upon confluence to perform an experiment, and each experiment was performed using HUVECs between P10 and P15.

\section{Cell-simulated ischemia/reperfusion model}

The I/R model was a modified version of a previously described method [5, 33, 34]. First, normal culture medium was replaced with serum-free DMEM prior to the start of the experiment. Following pregassing with $95 \% \mathrm{~N}_{2}$ and $5 \% \mathrm{CO}_{2}$ for a minimum of $5 \mathrm{~min}$, the ischemic buffer $\left(1 \mathrm{mM} \mathrm{NaH}_{2} \mathrm{PO}_{4}, 24 \mathrm{mM}\right.$ $\mathrm{NaHCO}_{3}, 2.5 \mathrm{mM} \mathrm{CaCl}_{2}, 118 \mathrm{mM} \mathrm{NaCl}, 16 \mathrm{mM} \mathrm{KCl}, 0.5 \mathrm{mM}$ sodium EDTA, $20 \mathrm{mM}$ sodium lactate, pH 6.8, $37^{\circ} \mathrm{C}$ ) was added to the cells, which were then placed in a sealed chamber containing the deoxygenation reagent, resulting in the consumption of $\mathrm{O}_{2}$ and the production of $\mathrm{CO}_{2}$. This Anaero-Pack system (Mitsubishi Gas Chemical Company, Inc.) created near-anaerobic conditions, with an $\mathrm{O}_{2}$ concentration of $<1 \%$ and a $\mathrm{CO}_{2}$ concentration of approximately $5 \%$ following a 1 -h incubation at $37^{\circ} \mathrm{C}$ [35]. To directly determine the efficacy of the equilibrating system, the $\mathrm{PO}_{2}$ of the medium was measured directly in a few of the experiments using a phosphorescence decay method [36, 37]. The actual supernatant $\mathrm{PO}_{2}$ values were $4.5 \pm 0.3 \mathrm{mmHg}$ (mean \pm SEM). Cells in a 24-well plate were treated with an ischemic buffer solution (1 mL/well) for $2 \mathrm{~h}$ prior to incubation in glucose-containing DMEM at $37^{\circ} \mathrm{C}$ in $95 \% \mathrm{O}_{2}$ and $5 \% \mathrm{CO}_{2}$ (reperfusion) for $24 \mathrm{~h}$.

Lentiviral transduction of HUVECS

HUVECs were transduced with LV-GFP lentivirus (Hanbio, Inc., Shanghai, CN) as previously described [5, 38-41]. Briefly, HUVECs (P3-5) were cultured in a 24-well plate $\left(1 \times 10^{4}\right.$ cells/well) in 10\% FBS in DMEM for $48 \mathrm{~h}$. The medium was then replaced with $1 \mathrm{~mL}$ of fresh medium and $8 \mu \mathrm{g} / \mathrm{mL}$ polybrene. Fifty microliters of lentivirus solution $\left(10^{7} \mathrm{IU} / \mathrm{mL}\right)$ was added to each well, and the plates were incubated at $37^{\circ} \mathrm{C}$ and $5 \%$ $\mathrm{CO}_{2}$ for $24 \mathrm{~h}$. Following incubation, the treatment medium was replaced with fresh $10 \% \mathrm{FBS}$ in DMEM, and the cells were cultured at $37^{\circ} \mathrm{C}$ and $5 \% \mathrm{CO}_{2}$ until $>50 \%$ confluence was reached. The transduced cells were selected using puromycin. For selection, the medium was replaced with $10 \mu \mathrm{g} / \mathrm{mL}$ puromycin in $10 \%$ FBS in DMEM, followed by culture at $37^{\circ} \mathrm{C}$ and $5 \% \mathrm{CO}_{2}$ for $24 \mathrm{~h}$. The cells were subsequently washed twice with fresh 10\% FBS in DMEM. Pure and transduced HUVEC cultures were expanded and stored in liquid nitrogen as previously described [42].

Tandem fluorescent-mRFP-GFP-MAP1LC3-adenovirus transduction of HUVECs

HUVECs were transfected with Tandem fluorescent-mRFP-GFP-MAP1LC3-adenovirus (Hanbio, Inc., Shanghai, $\mathrm{CN}$ ), a specific marker for autophagosome formation, to detect autophagy according to the manufacturer's instructions [43]. Five fields from three different cell preparations were selected. GFP and mRFP spots indicated by fluorescent puncta, as well as DAPI-stained nuclei, were counted manually. The number of spots per cell was determined by dividing the total number of spots by the number of nuclei in each field.

In vitro scratch assay

Cell migration in a 2D culture system was evaluated using an in vitro scratch assay [5]. Briefly, $1 \times$ $10^{5}$ HUVECs were seeded onto 24-well tissue culture plates and cultured in growth medium for $24 \mathrm{~h}$ until approximately $70-80 \%$ confluence. Using a sterile $200-\mu \mathrm{L}$ pipette tip, a straight line was carefully scratched into the monolayer across the center of the well in a single direction; the tip remained perpendicular to the plate bottom throughout this process. Similarly, a second straight line was scratched perpendicular to the first line to create a cross-shaped cellular gap in each well. Each well was washed twice with $1 \mathrm{~mL}$ of fresh growth medium to remove any detached cells. Digital images of the cell gaps were captured at different time points, and the gap widths were quantitatively evaluated using ImageJ software.

\section{Immunoblotting}

Immunoblotting was utilized as previously described [42] with minor modifications. The cells were washed with PBS and lysed using a Mammalian Cell Lysis kit (MCL1-1KT, Sigma-Aldrich) according to the manufacturer's instructions. Western blots were probed using different antibodies. The secondary antibodies used in this study were either goat anti-mouse or anti-rabbit IgG antibodies conjugated to alkaline phosphatase $(1: 5,000)$. The signals were detected via chemiluminescence (SuperSignal West Dura Chemiluminescent Substrate, Thermo Scientific). Each Western blot was repeated in three separate 


\section{Cellular Physiology Cell Physiol Biochem 2016;38:1575-1588 \\ \begin{tabular}{l|l} 
and Biochemistry Published online: April 18, 2016 & $\begin{array}{l}\text { Do } 2016 \text { The Author(s). Published by S. Karger AG, Basel } \\
\text { www.karger.com/cpb }\end{array}$
\end{tabular} \\ Zhu et al.: iNOS Activates HUVECs via Autophagy in I/R}

experiments. A single representative immunoblot for all related blots is presented in each figure. Densitometry was performed using ImageJ software (http://rsb.info.nih.gov/ij/), and the results for each of the repeated experiments were combined into one plot.

\section{MTT assay}

Cell viability was measured using the 3-(4,5-dmethylthiazol-2-yl)-2,5-diphenyl tetrazolium bromide (MTT) method [38]. Briefly, cells were collected and seeded onto 96-well plates. Different seeding densities were optimized at the beginning of the experiments. The cells were exposed to the I/R medium. Following incubation for different time periods (3-24 h), $20 \mu \mathrm{L}$ of MTT tetrazolium salt dissolved in Hank's balanced salt solution was added to each well at a final concentration of $5 \mu \mathrm{g} / \mathrm{mL}$, and the plates were incubated in a $\mathrm{CO}_{2}$ incubator for 1-4 h. Finally, the medium was aspirated from each well, and $200 \mu \mathrm{L}$ of dimethyl sulfoxide was added to dissolve the formazan crystals. The absorbance of each well was obtained using a microtiter plate spectrophotometer and reference wavelengths of $570 \mathrm{~nm}$ and $630 \mathrm{~nm}$. Each experiment was repeated at least three times.

\section{Hoechst staining}

To quantify the number of apoptotic cells, the cells were fixed and stained with $5 \mu \mathrm{M}$ Hoechst 33324 (Invitrogen) for $15 \mathrm{~min}$ at room temperature [44]. The morphological features of apoptosis (cell shrinkage, chromatin condensation, and fragmentation) were monitored using fluorescence microscopy (Nikon TE2000E microscope).

Statistics

The data are expressed as the mean \pm SEM. Unpaired numerical data were compared using either an unpaired t-test (two groups) or ANOVA (more than two groups); significance was established at $\mathrm{p}<0.05$.

\section{Results}

\section{I/R-mediated the up-regulation of iNOS in HUVECS}

Because iNOS has been shown to induce cell apoptosis in different conditions, we first examined the role of iNOS in the viability of HUVECs subjected to I/R. As shown in Fig. 1A, the exposure of HUVECs to I/R resulted in significantly decreased cell viability in a time-dependent manner, which was associated with increased iNOS expression (Fig. 1BC). Peak iNOS expression occurred at $3 \mathrm{~h}$ after reperfusion, which was confirmed using immunocytochemistry (Fig. 1D). Because iNOS expression increased following the exposure of HUVECs to I/R, we next attempted to determine the role of iNOS in the viability of HUVECs subjected to I/R. The pretreatment of HUVECs with a specific pharmacological inhibitor of iNOS, NG-Nitro-L-arginine Methyl Ester, Hydrochloride (L-NAME), for 1 hour prevented cell apoptosis 12 hours after exposure to I/R (Fig. 1E).

\section{iNOS induced HUVEC apoptosis via the caspase and Bcl/Bax pathways}

Because the caspase and Bcl-xl pathways play crucial roles in I/R-mediated apoptosis, we next examined the involvement of these pathways in iNOS-mediated cell apoptosis. First, we examined the effect of I/R on the expression of caspase 3 and caspase 9. As shown in Figs. 2A-D, exposure of HUVECs to I/R resulted in a transient increase in the expression of cleaved caspase 9 with a peak response at $6 \mathrm{~h}$, whereas I/R induced the sustained expression of cleaved caspase 3 with a peak response at $12 \mathrm{~h}$. In addition to the induction of the caspase pathways, I/R also induced activation of the Bcl/Bax pathway, as indicated by an increase in Bax and decrease in Bcl-xL expression, as well as an increased Bax/Bcl-xL ratio (Figs. 2E-F). We next attempted to determine the functional role of iNOS in the activation of apoptotic pathways induced by I/R. HUVECs were pretreated with an inhibitor specific for iNOS, L-NAME, prior to stimulation with I/R, and both iNOS expression and activation of Bax/Bcl-xL pathway was subsequently assessed. As shown in Figs. 2G-H, pretreatment of cells with L-NAME resulted in the amelioration of I/R-mediated induction of iNOS and 


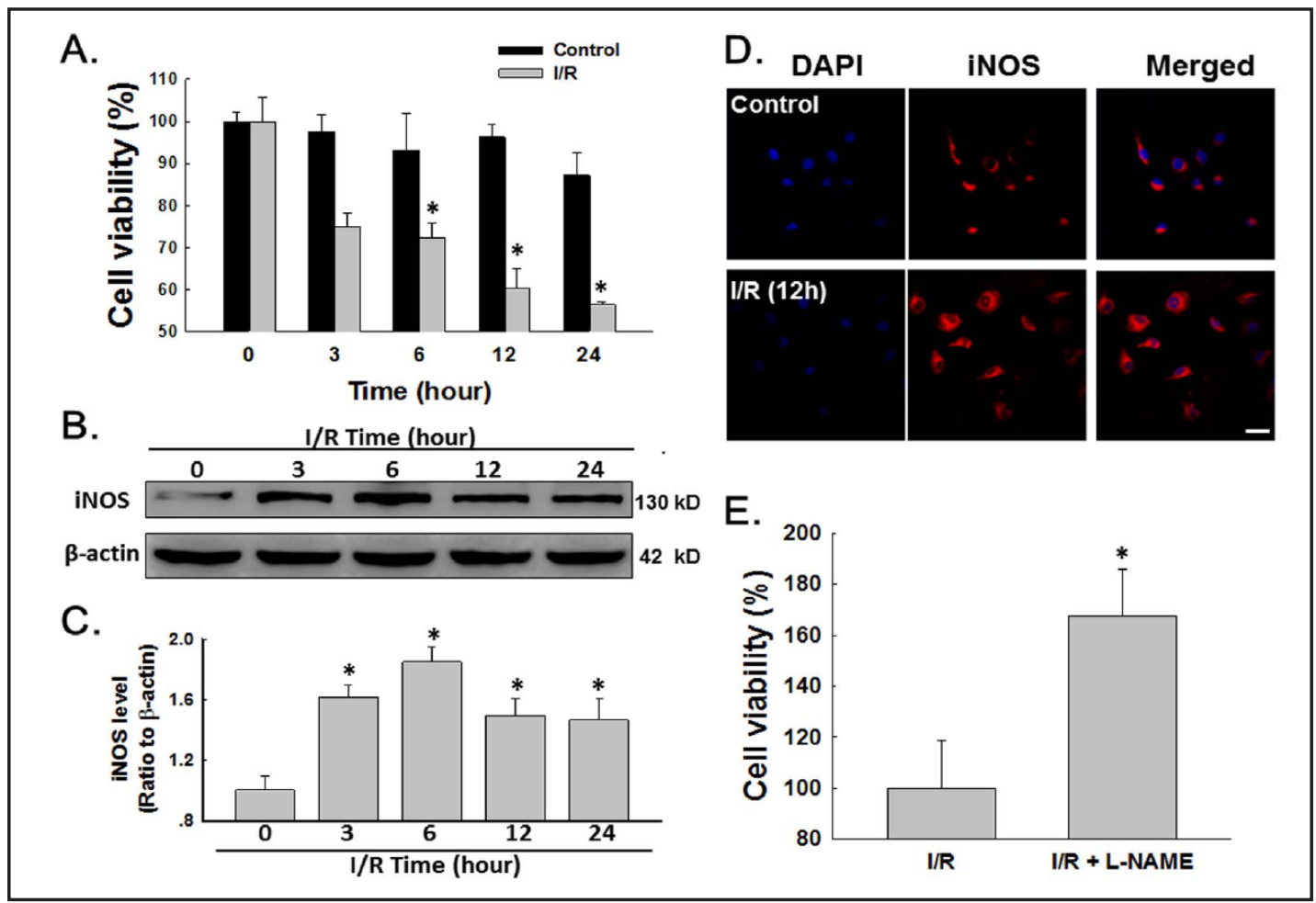

Fig. 1. I/R induced iNOS expression in HUVECs. (A) I/R decreased HUVEC viability in a time-dependent manner, as determined using the MTT assay; $p<0.05$ vs. the control group. (B) Representative blots demonstrating that I/R induced iNOS expression in a time-dependent manner. (C) Densitometric analyses of iNOS from four independent experiments; * $\mathrm{p}<0.05$ vs. the 0 -h group. (D) Representative immunocytochemical images showing that I/R induced iNOS expression in HUVECs. Scale bar, $50 \mu \mathrm{m}$. (E) Pretreatment of HUVECs with L-NAME attenuated the decrease in cell viability induced by I/R. * $p<0.05$ vs. the I/R group.

Bax/Bcl-xL. Interestingly, L-NAME did not inhibit the up-regulation of the caspase pathway (data not shown), which suggested that iNOS may mainly activate the Bax/Bcl-xL pathway. Taken together, these findings suggested the involvement of iNOS pathways in I/R-mediated activation of apoptosis pathways.

\section{Involvement of iNOS in I/R-mediated HUVEC migration}

Endothelial cell migration is a critical component of angiogenesis [5, 7]; thus, we next determined the role of iNOS in I/R-mediated migration. Using a scratch wound-healing assay, we determined that HUVEC migration increased following exposure to I/R, which was consistent with our previous findings (Figs. 2I-J) [5]. The increased migration induced by I/R in HUVECs was abolished by the pretreatment of cells with L-NAME, indicating the involvement of iNOS in this process.

\section{I/R-mediated the up-regulation of autophagy in HUVECS}

Mounting evidence has suggested an association between iNOS and autophagy in different settings $[45,46]$, and thus, we attempted to determine the role of iNOS and autophagy in the functional change of HUVECs subjected to I/R. As shown in Fig. 3A-D, the exposure of HUVECs to I/R significantly up-regulated the expression of the autophagy markers ATG5, LC3B and BECN in a time-dependent manner. The autophagic flux was further monitored in HUVECs transduced with tandem fluorescent-mRFP-GFP-MAP1LC3-adenovirus, a specific marker for autophagosome formation, which relies on the different nature of GFP and RFP fluorescence under acidic conditions [43]. GFP fluorescence is sensitive to the acidic condition of the lysosomal lumen, whereas RFP is relatively stable under acidic conditions. 


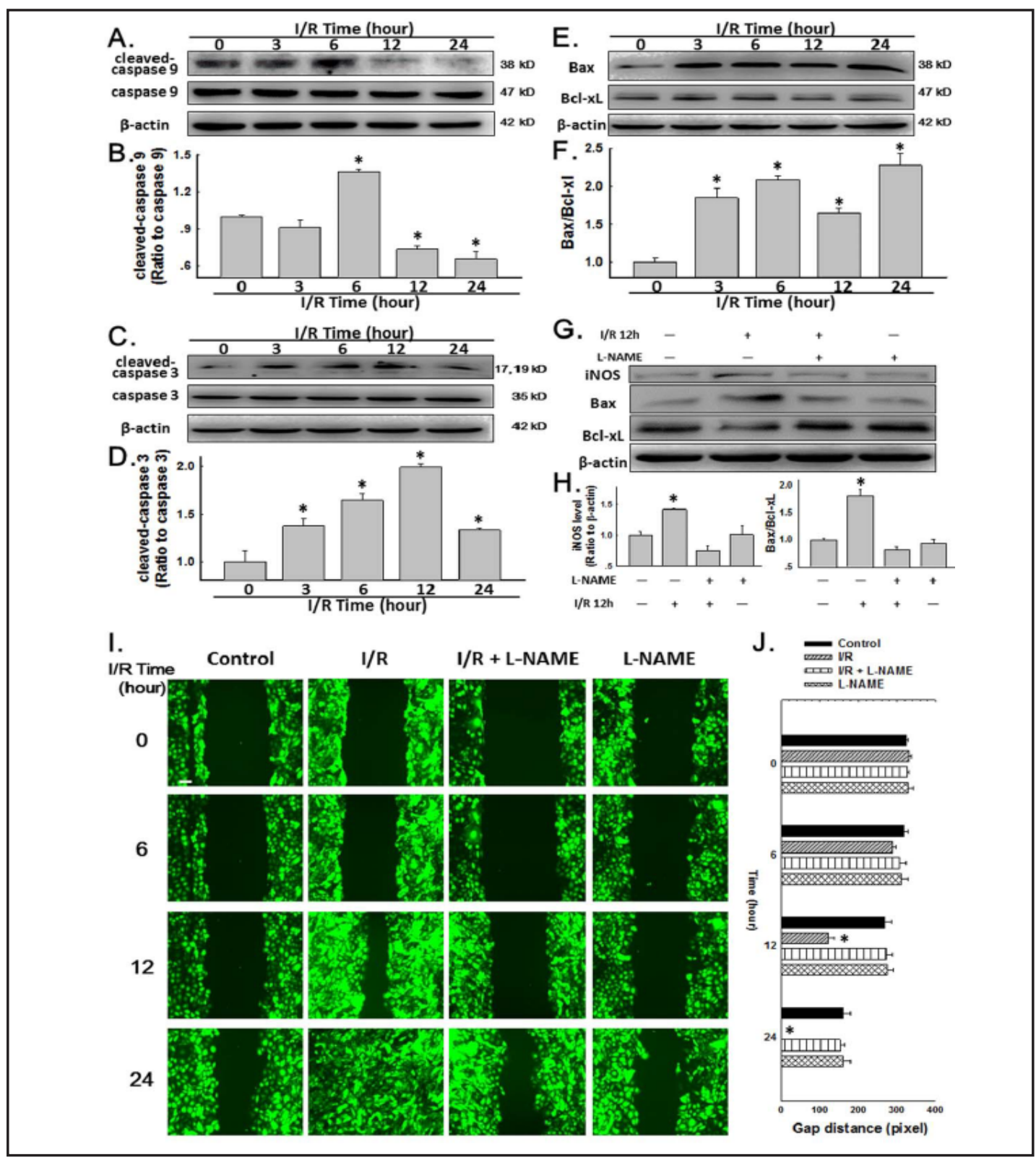

Fig. 2. I/R induced HUVEC cell apoptosis and migration. Representative blots demonstrating I/R-induced increase of cleaved-caspase 9 (A), cleaved-caspase 3 (C), Bax and decrease of Bcl-xL (E) in a time-dependent manner. Densitometric analyses of cleaved-caspase 9 (B), cleaved-caspase 3 (D) and Bax/Bcl-xL ratio (F) from four independent experiments are presented. ${ }^{*} \mathrm{p}<0.05$ vs. the 0 -h group. (G) Representative blots demonstrating that pretreatment of L-NAME on the expression of iNOS, Bax and Bcl-xL induced by I/R. (H) Densitometric analyses of iNOS, Bax/Bcl-xL ratio from four independent experiments. * $\mathrm{p}<0.05 \mathrm{vs}$. the I/R group. (I) Representative images depicting I/R-induced cell migration in monolayer cultured HUVECs was abolished by pretreatment with L-NAME. Scale bar, $80 \mu \mathrm{m}$. (J) Quantification of the scratch gap distance from six independent experiments is presented. ${ }^{*} \mathrm{p}<0.05$ vs. the corresponding time point in the control group.

Thus, the colocalization of GFP and RFP signals (yellow dots) indicates the phagophores or autophagosomes that have not fused with lysosomes, whereas RFP-only signals (red puncta) indicate the autolysosomes. As shown in Fig. 3E-G, I/R induced a significant increase in the number of yellow dots (Fig. 3G), with concomitantly enhanced elevation in RFP-only MAP1LC3 dots in the HUVECs of the I/R group (Fig. 3F-G). 


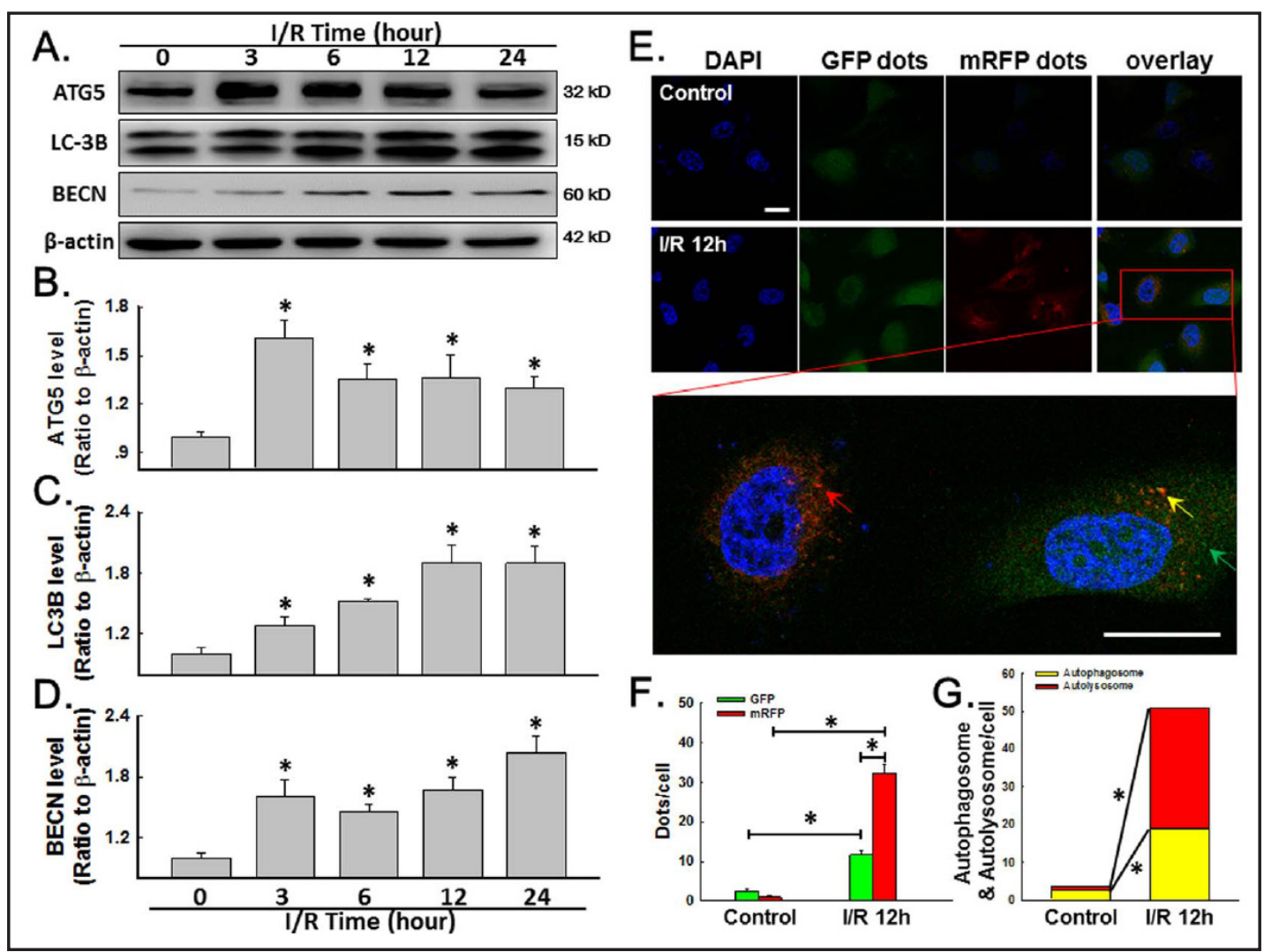

Fig. 3. I/R-induced autophagy in HUVECs. (A) Representative blots demonstrating that I/R-induced ATG5, LC-3B and BECN expression in time-dependent manner. Densitometric analyses of ATG5 (B), LC-3B (C) and BECN (D) from four independent experiments. * $p<0.05$ vs. the 0 -h group. (E) Representative images showing the effect of I/R on RFP- and GFP-MAP1LC3 puncta. Scale bar, $20 \mu \mathrm{m}$. (F) Quantification of the RFP- and GFP- MAP1LC3 puncta demonstrating I/R-induced autophagy. * $p<0.05$ vs. the control group. (G) Quantification of RFP-MAP1LC3 puncta and merged puncta demonstrating autophagy flux induced by I/R. * p $<0.05$ vs. the control group.

\section{Involvement of iNOS in I/R-mediated autophagy in HUVECS}

L-NAME was applied to determine the role of iNOS in autophagy induced by I/R. As shown in Fig. 4A-B, pretreatment of HUVECs with L-NAME significantly inhibited the elevated expression of ATG5, LC3B and BECN induced using I/R. Moreover, the up-regulation of both autophagosomes and autolysosomes was decreased by pretreatment with L-NAME, which suggested that increased autophagy was associated with I/R-induced iNOS in HUVECs. (Fig. 4C-E).

\section{Autophagy involvement in the cell migration induced by I/R in HUVECs}

To further understand the role of autophagy in I/R-induced HUVEC dysfunctions, the specific inhibitor of autophagy, 3-methyladenine (3-MA), which is a class III PI3K inhibitor that prevents autophagy at an early stage of autophagosome formation, was applied [25]. As shown in Fig. 5A-B, pretreatment with 3-MA for 1 hour attenuated the increase in cell migration induced by I/R in HUVECs, while 3-MA alone had no effect on cell migration compared with the control group. These data suggested that induction of autophagy plays a role in the up-regulation of migration after I/R treatment.

\section{Autophagy involved in apoptosis induced by I/R in HUVECs}

Next, we examined the effect of 3-MA on I/R-induced apoptosis. As shown in Fig. 6A-B, 3-MA pretreatment prevented the increased Bax/Bcl-xL ratio induced by I/R. In addition, the

\section{KARGER}




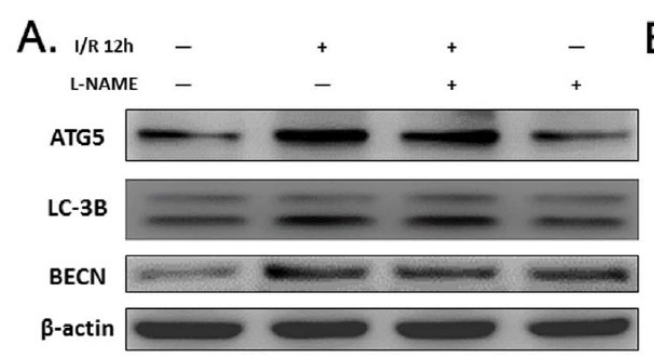

\section{B.}

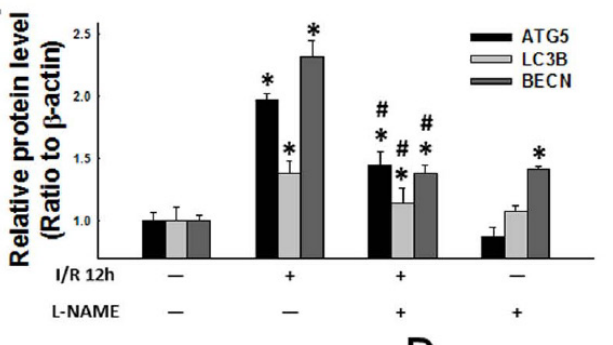

C.

DAPI

GFP dots

mRFP dots
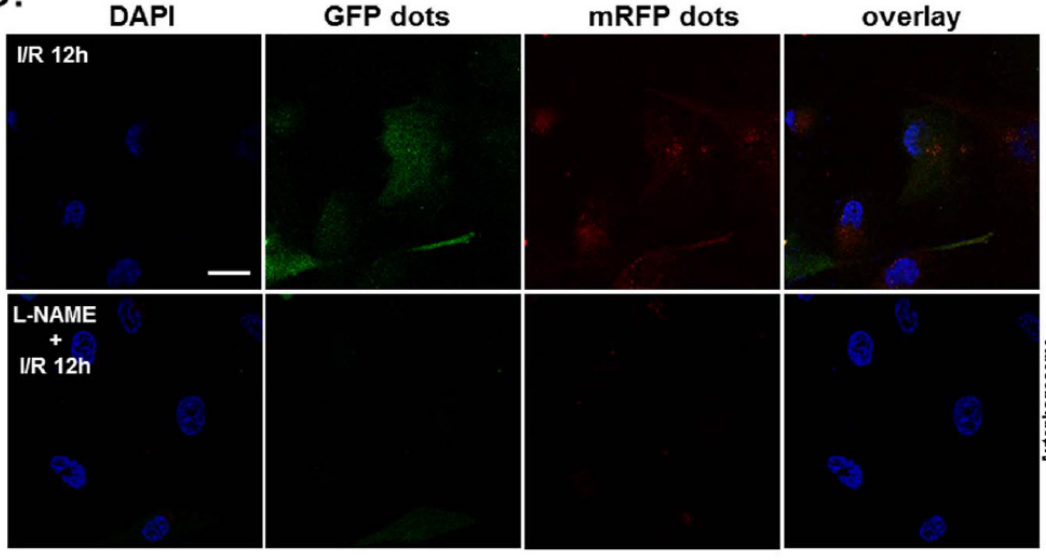

$\mathrm{D}_{\text {. }}$

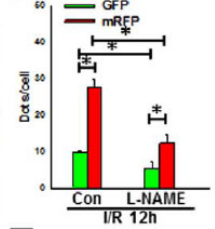

E.

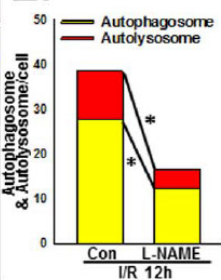

Fig. 4. Involvement of iNOS in I/R-induced autophagy in HUVECs. (A) Representative blots demonstrating that I/R-induced ATG5, LC-3B and BECN expression was abolished by pretreatment with L-NAME. (B) Densitometric analyses of ATG5, LC-3B and BECN from four independent experiments. ${ }^{*} \mathrm{p}<0.05$ vs. the control group. (C) Representative images showing pretreatment with L-NAME on I/R induced RFP- and GFP- MAP1LC3 puncta. Scale bar, $20 \mu \mathrm{m}$. (D) Quantification of RFP- and GFP- MAP1LC3 puncta demonstrating L-NAME attenuation of I/R-induced autophagy. * $\mathrm{p}<0.05$ vs. the control group. (E) Quantification of RFP-MAP1LC3 puncta and merged puncta demonstrating autophagy flux induced by I/R was attenuated by L-NAME. * $\mathrm{p}<0.05$ vs. the control group.

expression of cleaved caspase 9 induced by I/R was also ameliorated by pretreatment with 3-MA (Fig. 6C-D). Autophagy maintains the dynamic equilibrium, which contributes to the removal of toxic intracellular substances and promotes cell survival by providing recycled products. Under normal conditions, the application of 3-MA will disrupt the balance, which, in turn, affects cell survival [25]. Consistent with the finding in Fig. 6C-D, 3-MA alone exhibited an inhibitory effect on cell viability. However, the decreased activity of apoptosis pathways was accompanied by restored cell viability after I/R as a result of 3-MA pretreatment (Fig. $6 \mathrm{E}$ ). This finding was confirmed via cellular staining with Hoechst 33342, a nuclear dye that specifically stains nuclei. HUVECs in the control group were characterized by regular and round nuclei (Figs. 6F). In contrast, condensation and fragmentation of nuclei characteristic of apoptotic cells were evident in HUVECs subjected to I/R for $12 \mathrm{~h}$. Pretreatment of HUVECs with 3-MA significantly ameliorated I/R-induced cell death induced, as shown in Fig. 6F-G.

\section{Discussion}

While the timely reperfusion of acute ischemic myocardium is essential for myocardial salvage, which reduces infarct size, and prolongs life [47], reperfusion results in a unique form of myocardial damage that includes the formation of contraction bands in contractile proteins, calcific granules within mitochondria as well as cell swelling and disruption of 


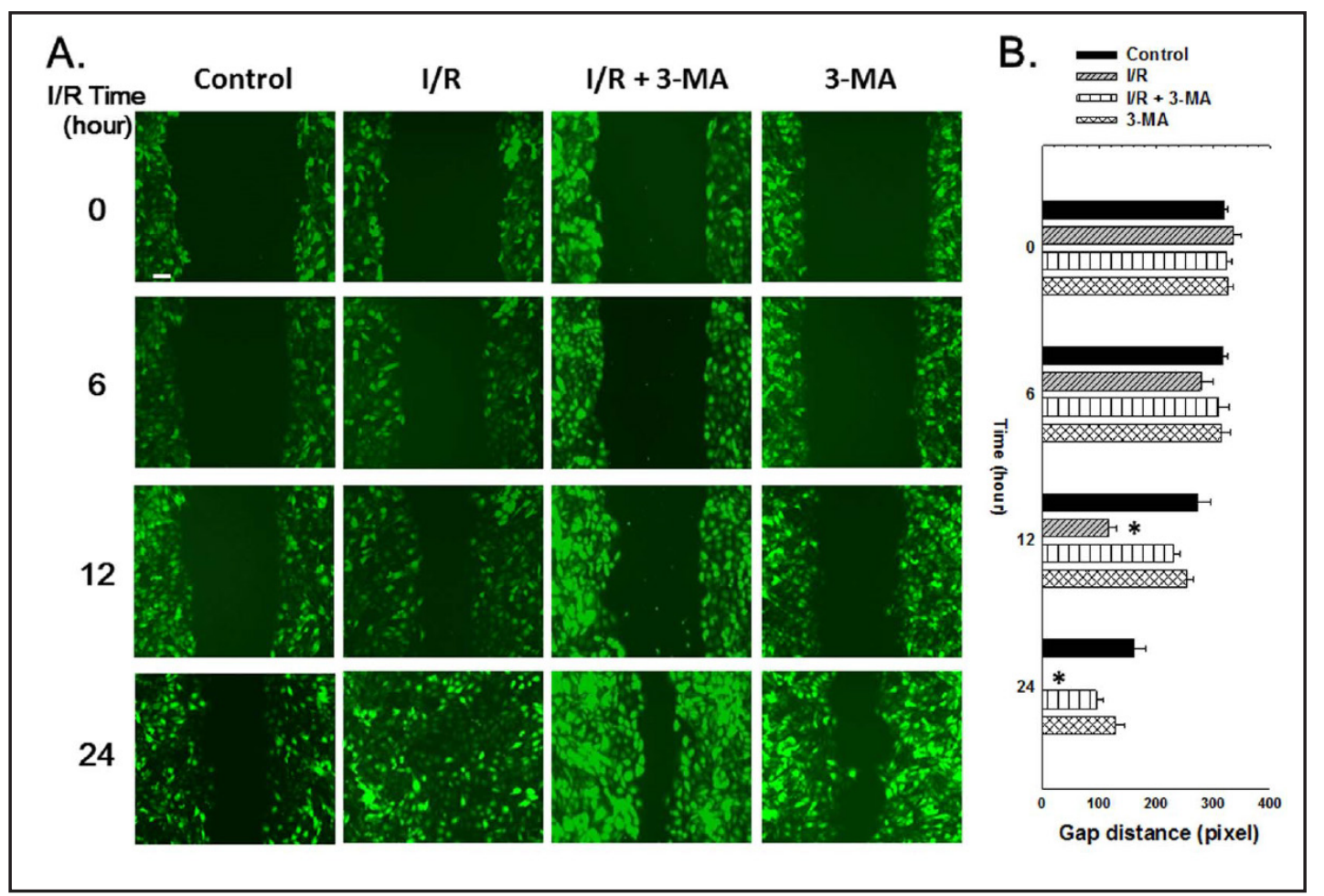

Fig. 5. Autophagy plays a role in I/R-induced HUVEC migration. (A) Representative images depicting attenuation of I/R-induced HUVEC migration by pretreatment with L-NAME. Scale bar, $80 \mu \mathrm{m}$. (B) Quantification of the scratch gap distance from six independent experiments is presented. ${ }^{*} \mathrm{p}<0.05 \mathrm{vs}$. the control group.

sarcoplasmic and mitochondrial membranes [48-50]. A number of mechanisms have been proposed to mediate reperfusion injury, such as cellular calcium loading, a lack of reflow phenomenon, impaired vascular relaxation, and the formation of oxygen radicals [43, 4850]. Impaired angiogenesis involves abnormalities of the microvasculature and contributes to the pathogenesis of I/R $[51,52]$. However, recent studies have suggested that ischemic myocardial disease may benefit from an enhancement of angiogenic processes and the establishment of collateral circulation $[2,3,53,54]$. In contrast to cardiomyocytes, the direct effect of I/R on endothelial cells has been less extensively researched. The aim of the current study was to investigate the role of endothelial cell-derived iNOS in cell activation and migration after I/R.

Endothelial cell dysfunction is involved in angiogenesis via different mechanisms, such as oxidative stress, inflammation and autophagy [7, 50,55], which affect endothelial cell activation, apoptosis, and migration [5]. Mounting evidence has suggested the role of oxidant and oxygen radical formation in this process, which serves as a critical central mechanism of post-ischemic injury $[50,56,57]$. Nitric oxide (NO), one of most important free radical and signaling molecules, plays diverse physiological and pathological roles. While low NO levels exert a number of regulatory and cytoprotective effects, higher levels are potentially toxic [50, $56,57]$. The up-regulation of iNOS, which is inducible, calcium-independent and involved in inflammation, results in excess NO production for prolonged periods of time, subsequently causing myocardial damage [57]. In the present study, we demonstrated that exposure of HUVECs to I/R resulted in the induction of iNOS, which induced cell apoptosis. Endothelial cell apoptosis was recently implicated in the initiation of angiogenesis and the regression of neo-vessels [14-16]. The functional significance of I/R-induced iNOS was examined in an in vitro model of cell migration. The exposure of HUVECs to I/R resulted in increased HUVEC migration. This effect was significantly inhibited by the specific iNOS blocker LNAME, thereby highlighting the role of iNOS in I/R-induced cell migration. These findings suggest

\section{KARGER}




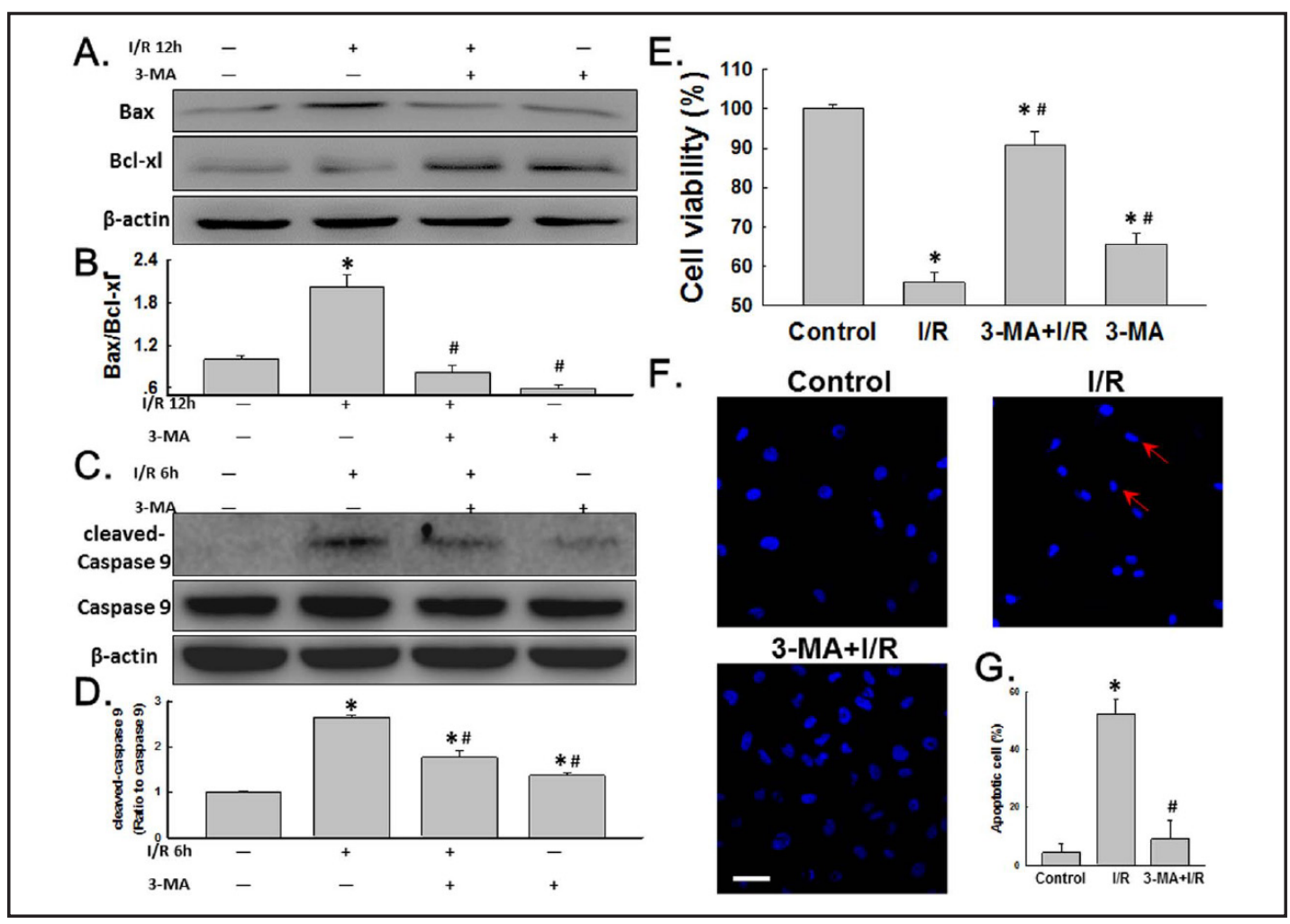

Fig. 6. Autophagy plays a role in I/R-induced apoptosis in HUVECs. (A) Representative blots demonstrating that I/R-induced Bax and Bcl-xL expression were abolished by pretreatment with 3-MA. (B) Densitometric analyses of the Bax/Bcl-xL ratio from four independent experiments. ${ }^{*} p<0.05$ vs. the control group. (C) Representative blots demonstrating that I/R-induced cleaved-caspase 9 expression was reduced by pretreatment with 3-MA. (D) Densitometric analyses of cleaved-caspase 9 from four independent experiments. * $p$ $<0.05$ vs. the control group. (E) Pretreatment of HUVECs with 3-MA attenuated the decrease in cell viability induced by I/R. * p $<0.05$ vs. the control group, \# p<0.05 vs. the I/R group. (F) Representative images of Hoechst 33342 staining depicting apoptosis of HUVECs induced by I/R was attenuated by 3-MA. Scale bar, $50 \mu \mathrm{m}$. (G) Percentages of apoptotic cells from six independent experiments are presented. * $p<0.05$ vs. the control group.

that iNOS may be critical in the regulation of I/R injury not only in cardiomyocytes but also in endothelial cells.

To obtain a deeper understanding of the mechanisms underlying I/R-mediated induction of iNOS expression on endothelial cell dysfunction, the roles of autophagy were assessed. Previous data from our lab have suggested that MCPIP1 plays an important role in I/R-induced cell migration and apoptosis [5]. Interestingly, MCPIP1 has recently been demonstrated to have a role in the regulation of autophagy and further promote cell apoptosis $[58,59]$. Consistent with our finding, in preclinical models of heart disease, cardiomyocyte autophagic flux is activated, while its role in disease pathogenesis is the subject of intense investigation to define the mechanism [60]. Moreover, the reduction of Beclin1 expression in cardiac myocytes by RNAi reduces I/R-induced autophagy, and this reduction is associated with enhanced cell survival [61]. In the current study, I/R induced a slight but significant increase in expression of the autophagy markers ATG5, LC3B and BECN, which suggests that autophagy is at least involved in I/R-induced injury. However, whether iNOS-induced endothelial cell dysfunction occurs via autophagy remains unclear. In the current study, we demonstrated that L-NAME blocked the expression of ATG5, LC3B and BECN, which are markers of autophagy. Furthermore, the autophagic flux induced by I/R was also blocked

\section{KARGER}




\section{Cellular Physiology Cell Physiol Biochem 2016;38:1575-1588

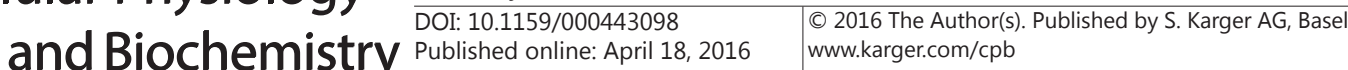 \\ Zhu et al.: iNOS Activates HUVECs via Autophagy in I/R}

by pretreatment of HUVECs with L-NAME, which strongly indicated that iNOS induced cell apoptosis via autophagy. To further understand the role of autophagy in endothelial cell dysfunction, the pharmacological inhibitor of autophagy, 3-MA, was applied. 3-MA not only abolished the increase in cell migration but also rescued cells from I/R-induced apoptosis. These findings indicated an association between iNOS and autophagy in endothelial cell dysfunction induced by I/R.

In summary, our findings have identified a new role for iNOS in endothelial cells that activates autophagy in response to I/R. Furthermore, I/R-mediated expression of iNOS played a vital role in cell migration and apoptosis, resulting in increased angiogenesis and apoptosis during the late stages of I/R. These findings have implications for I/R among individuals with heart failure. Understanding the regulation of iNOS and autophagy may aid in the development of adjunct therapeutic strategies in the treatment of individuals with $\mathrm{I} / \mathrm{R}$.

\section{Acknowledgments}

This study was partially supported by the resources and facilities of the core lab of the Medical School of Southeast University. BTZ was supported by grants obtained from the Natural Science Foundation of Jiangsu Province, China (No. BK20141497). JC was supported by grants obtained from the National Natural Science Foundation of China (81473263) and Natural Science Foundation of Jiangsu Province, China (No. BK20141347).

\section{Disclosures Statement}

The authors have no competing financial interests to disclose.

\section{References}

1 Liu Y, Yang H, Song L, Li N, Han QY, Tian C, Gao E, Du J, Xia YL, Li HH: Aggf1 protects from myocardial ischemia/reperfusion injury by regulating myocardial apoptosis and angiogenesis. Apoptosis 2014;19:1254-1268.

2 Altamirano F, Wang ZV, Hill JA: Cardioprotection in ischemia/reperfusion injury: Novel mechanisms and clinical translation. J Physiol 2015;593:3773-3788.

3 Song MA, Paradis AN, Gay MS, Shin J, Zhang L: Differential expression of micrornas in ischemic heart disease. Drug Discov Today 2015;20:223-235.

4 Yu D, Li M, Tian Y, Liu J, Shang J: Luteolin inhibits ros-activated mapk pathway in myocardial ischemia/ reperfusion injury. Life Sci 2015;122:15-25.

5 Zhu T, Yao Q, Hu X, Chen C, Yao H, Chao J: The role of mcpip1 in ischemia/reperfusion injury-induced huvec migration and apoptosis. Cell Physiol Biochem 2015;37:577-591.

6 Luo T, Chen B, Zhao Z, He N, Zeng Z, Wu B, Fukushima Y, Dai M, Huang Q, Xu D, Bin J, Kitakaze M, Liao Y: Histamine h2 receptor activation exacerbates myocardial ischemia/reperfusion injury by disturbing mitochondrial and endothelial function. Basic Res Cardiol 2013;108:342.

7 Niu J, Azfer A, Zhelyabovska O, Fatma S, Kolattukudy PE: Monocyte chemotactic protein (mcp)-1 promotes angiogenesis via a novel transcription factor, mcp-1-induced protein (mcpip). J Biol Chem 2008;283:14542-14551.

8 Zhang X, Liang X, Lin X, Zhang S, Huang Z, Chen C, Guo Y, Xuan F, Xu X, Huang R: Mechanism of the protective effect of yulangsan flavonoid on myocardial ischemia/reperfusion injury in rats. Cell Physiol Biochem 2014;34:1050-1062.

9 Calvillo L, Vanoli E, Andreoli E, Besana A, Omodeo E, Gnecchi M, Zerbi P, Vago G, Busca G, Schwartz PJ: Vagal stimulation, through its nicotinic action, limits infarct size and the inflammatory response to myocardial ischemia and reperfusion. J Cardiovasc Pharmacol 2011;58:500-507. 


\section{Cellular Physiology Cell Physiol Biochem 2016;38:1575-1588

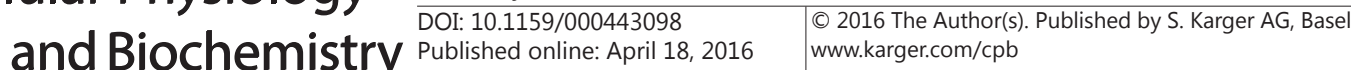 \\ Zhu et al.: iNOS Activates HUVECs via Autophagy in I/R}

10 Charo IF, Taubman MB: Chemokines in the pathogenesis of vascular disease. Circ Res 2004;95:858-866.

11 Ito WD, Arras M, Winkler B, Scholz D, Schaper J, Schaper W: Monocyte chemotactic protein-1 increases collateral and peripheral conductance after femoral artery occlusion. Circ Res 1997;80:829-837.

12 Carmeliet P: Angiogenesis in life, disease and medicine. Nature 2005;438:932-936.

13 Wang X, Zhang Y, Zhang W, Liu H, Zhou Z, Dai X, Cheng Y, Fang S, Yao H, Chao J: Mcpip1 regulates alveolar macrophage apoptosis and pulmonary fibroblast activation after in vitro exposure to silica. Toxicol Sci 2016; DOI: $10.1093 /$ toxsci/kfw029

14 Peters K, Troyer D, Kummer S, Kirkpatrick CJ, Rauterberg J: Apoptosis causes lumen formation during angiogenesis in vitro. Microvasc Res 2002;64:334-338.

15 Tertemiz F, Kayisli UA, Arici A, Demir R: Apoptosis contributes to vascular lumen formation and vascular branching in human placental vasculogenesis. Biol Reprod 2005;72:727-735.

16 Duval H, Harris M, Li J, Johnson N, Print C: New insights into the function and regulation of endothelial cell apoptosis. Angiogenesis 2003;6:171-183.

17 Cho SO, Lim JW, Kim H: Red ginseng extract inhibits the expression of mcp-1 and inos in helicobacter pylori-infected gastric epithelial cells by suppressing the activation of nadph oxidase and jak2/stat3. J Ethnopharmacol 2013;150:761-764.

18 Su Z, Ye J, Qin Z, Ding X: Protective effects of madecassoside against doxorubicin induced nephrotoxicity in vivo and in vitro. Sci Rep 2015;5:18314.

19 Chen H, Xu Y, Wang J, Zhao W, Ruan H: Baicalin ameliorates isoproterenol-induced acute myocardial infarction through inos, inflammation and oxidative stress in rat. Int J Clin Exp Pathol 2015;8:1013910147.

20 Martinez-Gonzalez J, Alfon J, Berrozpe M, Badimon L: Hmg-coa reductase inhibitors reduce vascular monocyte chemotactic protein-1 expression in early lesions from hypercholesterolemic swine independently of their effect on plasma cholesterol levels. Atherosclerosis 2001;159:27-33.

21 Khanna A, Guo M, Mehra M, Royal W, 3rd: Inflammation and oxidative stress induced by cigarette smoke in lewis rat brains. J Neuroimmunol 2013;254:69-75.

22 Zhang Y, Guo W, Wen Y, Xiong Q, Liu H, Wu J, Zou Y, Zhu Y: Scm-198 attenuates early atherosclerotic lesions in hypercholesterolemic rabbits via modulation of the inflammatory and oxidative stress pathways. Atherosclerosis 2012;224:43-50.

23 Rabinowitz JD, White E: Autophagy and metabolism. Science 2010;330:1344-1348.

24 Iwata J, Ezaki J, Komatsu M, Yokota S, Ueno T, Tanida I, Chiba T, Tanaka K, Kominami E: Excess peroxisomes are degraded by autophagic machinery in mammals. J Biol Chem 2006;281:4035-4041.

25 Kim PK, Hailey DW, Mullen RT, Lippincott-Schwartz J: Ubiquitin signals autophagic degradation of cytosolic proteins and peroxisomes. Proc Natl Acad Sci USA 2008;105:20567-20574.

26 Azad MB, Chen Y, Henson ES, Cizeau J, McMillan-Ward E, Israels SJ, Gibson SB: Hypoxia induces autophagic cell death in apoptosis-competent cells through a mechanism involving bnip3. Autophagy 2008;4:195-204.

27 Yousefi S, Perozzo R, Schmid I, Ziemiecki A, Schaffner T, Scapozza L, Brunner T, Simon HU: Calpainmediated cleavage of atg5 switches autophagy to apoptosis. Nat Cell Biol 2006;8:1124-1132.

28 Green DR, Galluzzi L, Kroemer G: Mitochondria and the autophagy-inflammation-cell death axis in organismal aging. Science 2011;333:1109-1112.

29 Levine B, Mizushima N, Virgin HW: Autophagy in immunity and inflammation. Nature 2011;469:323-335.

30 Surico D, Farruggio S, Marotta P, Raina G, Mary D, Surico N, Vacca G, Grossini E: Human chorionic gonadotropin protects vascular endothelial cells from oxidative stress by apoptosis inhibition, cell survival signalling activation and mitochondrial function protection. Cell Physiol Biochem 2015;36:2108-2120.

31 Hsieh MJ, Lin CW, Chiou HL, Yang SF, Chen MK: Dehydroandrographolide, an inos inhibitor, extracted from andrographis paniculata (burm.F.) nees, induces autophagy in human oral cancer cells. Oncotarget 2015;6:30831-30849.

32 Kandemir FM, Ozkaraca M, Yildirim BA, Hanedan B, Kirbas A, Kilic K, Aktas E, Benzer F: Rutin attenuates gentamicin-induced renal damage by reducing oxidative stress, inflammation, apoptosis, and autophagy in rats. Ren Fail 2015;37:518-525.

33 Han L, Xu C, Jiang C, Li H, Zhang W, Zhao Y, Zhang L, Zhang Y, Zhao W, Yang B: Effects of polyamines on apoptosis induced by simulated ischemia/reperfusion injury in cultured neonatal rat cardiomyocytes. Cell Biol Int 2007;31:1345-1352. 


\section{Cellular Physiology Cell Physiol Biochem 2016;38:1575-1588 \begin{tabular}{l|l} 
and Biochemistry Published online: April 18, 2016 & $\begin{array}{l}\text { D) } 2016 \text { The Author(s). Published by S. Karger AG, Basel } \\
\text { www.karger.com/cpb }\end{array}$ \\
\hline
\end{tabular} \\ Zhu et al.: iNOS Activates HUVECs via Autophagy in $\mathrm{I} / \mathrm{R}$}

34 Yan L, Zhu TB, Wang LS, Pan SY, Tao ZX, Yang Z, Cao K, Huang J: Inhibitory effect of hepatocyte growth factor on cardiomyocytes apoptosis is partly related to reduced calcium sensing receptor expression during a model of simulated ischemia/reperfusion. Mol Biol Rep 2011;38:2695-2701.

35 Van Horn K, Toth C: Evaluation of the anaeropack campylo system for growth of microaerophilic bacteria. J Clin Microbiol 1999;37:2376-2377.

36 Lo LW, Koch CJ, Wilson DF: Calibration of oxygen-dependent quenching of the phosphorescence of pdmeso-tetra (4-carboxyphenyl) porphine: A phosphor with general application for measuring oxygen concentration in biological systems. Anal Biochem 1996;236:153-160.

37 Chao J, Wood JG, Blanco VG, Gonzalez NC: The systemic inflammation of alveolar hypoxia is initiated by alveolar macrophage-borne mediator(s). Am J Respir Cell Mol Biol 2009;41:573-582.

38 Liu X, Fang S, Liu H, Wang X, Dai X, Yin Q, Yun T, Wang W, Zhang Y, Liao H, Zhang W, Yao H, Chao J: Role of human pulmonary fibroblast-derived mcp-1 in cell activation and migration in experimental silicosis. Toxicol Appl Pharmacol 2015;288:152-160.

39 Chao J, Pena T, Heimann DG, Hansen C, Doyle DA, Yanala UR, Guenther TM, Carlson MA: Expression of green fluorescent protein in human foreskin fibroblasts for use in $2 \mathrm{~d}$ and $3 \mathrm{~d}$ culture models. Wound Repair Regen 2014;22:134-140.

40 Wang W, Liu H, Dai X, Fang S, Wang X, Zhang Y, Yao H, Zhang X, Chao J: P53/puma expression in human pulmonary fibroblasts mediates cell activation and migration in silicosis. Sci Rep 2015;5:16900.

41 Liu H, Dai X, Cheng Y, Fang S, Zhang Y, Wang X, Zhang W, Liao H, Yao H, Chao J: Mcpip1 mediates silica-induced cell migration in human pulmonary fibroblasts. Am J Physiol Lung Cell Mol Physiol 2015;310:L121-132.

42 Carlson MA, Longaker MT, Thompson JS: Modulation of fak, akt, and p53 by stress release of the fibroblastpopulated collagen matrix. J Surg Res 2004;120:171-177.

43 Hariharan N, Zhai P, Sadoshima J: Oxidative stress stimulates autophagic flux during ischemia/reperfusion. Antioxid Redox Signal 2011;14:2179-2190.

44 Li HY, Cui XY, Wu W, Yu FY, Yao HR, Liu Q, Song EW, Chen JQ: Pyk2 and src mediate signaling to ccl18induced breast cancer metastasis. J Cell Biochem 2014;115:596-603.

45 Talero E, Garcia-Maurino S, Motilva V: Melatonin, autophagy and intestinal bowel disease. Curr Pharm Des 2014;20:4816-4827.

46 Sarkar S, Korolchuk VI, Renna M, Imarisio S, Fleming A, Williams A, Garcia-Arencibia M, Rose C, Luo S, Underwood BR, Kroemer G, O'Kane CJ, Rubinsztein DC: Complex inhibitory effects of nitric oxide on autophagy. Mol Cell 2011;43:19-32.

47 Keeley EC, Boura JA, Grines CL: Primary angioplasty versus intravenous thrombolytic therapy for acute myocardial infarction: A quantitative review of 23 randomised trials. Lancet 2003;361:13-20.

48 Yellon DM, Hausenloy DJ: Myocardial reperfusion injury. N Engl J Med 2007;357:1121-1135.

49 Schafer C, Ladilov Y, Inserte J, Schafer M, Haffner S, Garcia-Dorado D, Piper HM: Role of the reverse mode of the na+/ca2+ exchanger in reoxygenation-induced cardiomyocyte injury. Cardiovasc Res 2001;51:241-250.

50 Zweier JL, Talukder MA: The role of oxidants and free radicals in reperfusion injury. Cardiovasc Res 2006;70:181-190.

51 Shimizu T, Okamoto H, Chiba S, Matsui Y, Sugawara T, Akino M, Nan J, Kumamoto H, Onozuka H, Mikami T, Kitabatake A: Vegf-mediated angiogenesis is impaired by angiotensin type 1 receptor blockade in cardiomyopathic hamster hearts. Cardiovasc Res 2003;58:203-212.

52 Ibanez B, Heusch G, Ovize M, Van de Werf F: Evolving therapies for myocardial ischemia/reperfusion injury. J Am Coll Cardiol 2015;65:1454-1471.

53 Yang B, Yang J, Bai J, Pu P, Liu J, Wang F, Ruan B: Suv39h1 protects from myocardial ischemia-reperfusion injury in diabetic rats. Cell Physiol Biochem 2014;33:1176-1185.

54 Li Q Wang F, Zhang YM, Zhou JJ, Zhang Y: Activation of cannabinoid type 2 receptor by jwh133 protects heart against ischemia/reperfusion-induced apoptosis. Cell Physiol Biochem 2013;31:693-702.

55 Frangogiannis NG, Smith CW, Entman ML: The inflammatory response in myocardial infarction. Cardiovasc Res 2002;53:31-47.

56 Panaro MA, Brandonisio O, Acquafredda A, Sisto M, Mitolo V: Evidences for inos expression and nitric oxide production in the human macrophages. Curr Drug Targets Immune Endocr Metabol Disord 2003;3:210221. 


\section{Cellular Physiology Cell Physiol Biochem 2016;38:1575-1588 \begin{tabular}{l|l|l} 
DOI: 10.1159/000443098 & $\begin{array}{l}\text { O 2016 The Author(s). Published by S. Karger AG, Basel } \\
\text { www.karger.com/cpb }\end{array}$
\end{tabular} \\ Zhu et al.: iNOS Activates HUVECs via Autophagy in I/R}

57 Wildhirt SM, Weismueller S, Schulze C, Conrad N, Kornberg A, Reichart B: Inducible nitric oxide synthase activation after ischemia/reperfusion contributes to myocardial dysfunction and extent of infarct size in rabbits: Evidence for a late phase of nitric oxide-mediated reperfusion injury. Cardiovasc Res 1999;43:698711.

58 Younce CW, Kolattukudy PE: Mcp-1 causes cardiomyoblast death via autophagy resulting from er stress caused by oxidative stress generated by inducing a novel zinc-finger protein, mcpip. Biochem J 2010;426:43-53.

59 Roy A, Kolattukudy PE: Monocyte chemotactic protein-induced protein (mcpip) promotes inflammatory angiogenesis via sequential induction of oxidative stress, endoplasmic reticulum stress and autophagy. Cell Signal 2012;24:2123-2131.

60 Xie M, Morales CR, Lavandero S, Hill JA: Tuning flux: Autophagy as a target of heart disease therapy. Curr Opin Cardiol 2011;26:216-222.

61 Valentim L, Laurence KM, Townsend PA, Carroll CJ, Soond S, Scarabelli TM, Knight RA, Latchman DS, Stephanou A: Urocortin inhibits beclin1-mediated autophagic cell death in cardiac myocytes exposed to ischaemia/reperfusion injury. J Mol Cell Cardiol 2006;40:846-852. 